\title{
Second Order Statistical Moments of the Power Spectrum of Ionospheric Scintillation
}

\author{
Jandieri George ${ }^{1,}$, , Diasamidze Zhuzhuna ${ }^{2}$, Diazamidze Mzia ${ }^{3}$, Takidze Irma ${ }^{3}$ \\ ${ }^{1}$ Physics Dept., Georgian Technical University, GTU, Tbilisi, Georgia \\ ${ }^{2}$ Batumi Shota Rustaveli State University, BASU, Batumi, Georgia \\ ${ }^{3}$ Batumi State Maritime Academy, Batumi, Georgia \\ Email address: \\ georgejandieri7@gmail.com (J. George), zhuzhuna.diasamidze@gmail.com (D. Zhuzhuna), mzia.diasamidze@gmail.com (D. Mzia) \\ ${ }^{*}$ Corresponding author
}

\section{To cite this article:}

Jandieri George, Diasamidze Zhuzhuna, Diazamidze Mzia, Takidze Irma. Second Order Statistical Moments of the Power Spectrum of Ionospheric Scintillation. Earth Sciences. Vol. 6, No. 6, 2017, pp. 142-148. doi: 10.11648/j.earth.20170606.16

Received: April 6, 2017; Accepted: April 17, 2017; Published: October 31, 2017

\begin{abstract}
Paper considers some aspects of the relationship between scintillation level of scattered radiation and anisotropic plasma irregularities in the collision turbulent magnetized plasma. Analytical calculations are carried out using modify smooth perturbation method taking into account both diffraction effects and polarization coefficients of both ordinary and extraordinary waves. Correlation function and the wave structure function of the phase fluctuations are obtained for arbitrary correlation function of electron density fluctuations. Analytical and numerical calculations of the second order statistical moments are carried out for the anisotropic Gaussian correlation function taking into account both anisotropy factor and the inclination angle of the elongated plasma irregularities with respect to the external magnetic field. Scintillation level and the power spectrum of the intensity fluctuations are analyzed for different parameters characterizing anisotropic irregularities.
\end{abstract}

Keywords: Turbulence, Ionosphere, Irregularities, Magnetized Plasma

\section{Introduction}

Many excellent reviews and books of scintillation theory and observations $[1,2]$ have been published. A high priority given to the ionospheric scintillation comes from its significant impact on satellite radio communications. Scintillation of satellite radio signals is a consequence of the existence of random electron density fluctuations within the ionosphere.

The irregularities have different spatial sizes and usually are elongated in the magnetic field direction. The connection between phase and amplitude fluctuations and the irregularities causing them has been studied in [3]. Peculiarities of the second order statistical moments of scattered radiation in collision magnetized plasma using the ray (-optics) approximation have been investigated in [4]. Scintillation spectra of scattered ordinary and extraordinary waves in the turbulent collisionless magnetized plasma have been considered in [5]. Analytical and numerical calculations were carried out for the anisotropic Gaussian spectrum taking into account diffraction effects. Statistical moments of the spatial power spectrum of multiple scattered ordinary and extraordinary waves in the turbulent collision magnetized plasma with aligned anisotropic electron density irregularities were investigated in $[6,7]$ using the modify smooth perturbation method.

In this paper we consider some aspects of the relationship between scintillation and ionospheric irregularities. Analytical and numerical calculations are carried out for the anisotropic Gaussian spectral function containing important experimentally measured parameters: anisotropy factor and slope angle of elongated plasma irregularities with respect to the lines of force of geomagnetic field. Scintillation level is estimated for different anisotropy factor and the angle of inclination of elongated irregularities with respect to the external magnetic field. Numerical calculations are based on the experimental data. 


\section{Statistical Moments of Scattered Radiation in the Ionospheric Plasma}

Electric field in the turbulent collision plasma satisfies the wave equation:

$$
\left(\frac{\partial^{2}}{\partial x_{i} \partial x_{j}}-\Delta \delta_{i j}-k_{0}^{2} \varepsilon_{i j}(\mathbf{r})\right) \mathbf{E}_{\mathbf{j}}(\mathbf{r})=0
$$

with the components of the dielectric permittivity [8]:

$$
\begin{gathered}
\varepsilon_{x x}=1-\frac{\mathrm{v} g}{g^{2}-u}, \varepsilon_{x y}=-\varepsilon_{y x}=i \frac{\mathrm{v} \sqrt{u} \cos \alpha}{g^{2}-u}, \\
\varepsilon_{x z}=-\varepsilon_{z x}=-i \frac{\mathrm{v} \sqrt{u} \sin \alpha}{g^{2}-u}, \varepsilon_{y y}=1-\frac{\mathrm{v}\left(g^{2}-u \sin ^{2} \alpha\right)}{g\left(g^{2}-u\right)}, \\
\varepsilon_{y z}=\varepsilon_{z y}=\frac{u \mathrm{v} \sin \alpha \cos \alpha}{g\left(g^{2}-u\right)}, \varepsilon_{z z}=1-\frac{\mathrm{v}\left(g^{2}-u \cos ^{2} \alpha\right)}{g\left(g^{2}-u\right)} .
\end{gathered}
$$

where: $\omega_{p}(\mathbf{r})=\left[4 \pi N(\mathbf{r}) e^{2} / m\right]^{1 / 2}$ is the plasma frequency, $N(\mathbf{r})$ is the electron concentration, $\mathrm{v}(\mathbf{r})=\omega_{p}^{2}(\mathbf{r}) / \omega^{2}$ and $u(\mathbf{r})=\left(e H_{0}(\mathbf{r}) / m c \omega\right)^{2}$ are the magneto-ionic paramters, e and $\mathrm{m}$ are the charge and the mass of electron, $g=1-i s$, $s=v_{\text {eff }} / \omega, \quad v_{e f f}=v_{e i}+v_{e n}$ is the effective collision fre-quency of electrons with other plasma particles, $\alpha$ is the angle between the $\mathrm{Z}$-axis (the direction of the wave propa-gation) and the static external magnetic field $\mathbf{H}_{\mathbf{0}}$ in the YOZ principle plane.

Polarization coefficients in the turbulent collision magnetized plasma are [8]:

$$
\frac{<E_{z}>_{1,2}}{<E_{x}>_{1,2}}=i \Gamma_{j}^{\prime \prime}+s \Gamma_{j}^{\prime}, \frac{<E_{y}>_{1,2}}{<E_{x}>_{1,2}}=-i \mathrm{P}_{j}^{\prime \prime}-s \mathrm{P}_{j}^{\prime},
$$

where: $\mathrm{P}_{j}^{\prime \prime}=\frac{2 \sqrt{u_{L}}}{u_{T} \mp \sqrt{a_{*}}}\left[(1-\mathrm{v}) \mp 2 s^{2} \frac{u_{L}(5-4 \mathrm{v})}{a_{*}\left(u_{T} \mp \sqrt{a_{*}}\right)}\right]$,

$$
\begin{aligned}
& a_{*}=u_{T}^{2}+4 u_{L}(1-\mathrm{v})^{2}, D=\frac{4 u_{L}(1-\mathrm{v})^{2}}{\sqrt{a_{*}}\left(u_{T} \mp \sqrt{a_{*}}\right)}, \\
& \mathrm{P}_{j}^{\prime}=\frac{2 \sqrt{u_{L}}}{u_{T} \mp \sqrt{a_{*}}}(1 \pm D) \pm 2 s^{2} \frac{\sqrt{u_{L}}}{\sqrt{a_{*}}\left(u_{T} \mp \sqrt{a_{*}}\right)} . \\
& \cdot\left[\frac{D\left(u_{T} \mp \sqrt{a_{*}}\right)}{\sqrt{a_{*}}}-(1 \pm D)\right], t_{0}=1-u-\mathrm{v}+\mathrm{v} u_{L}, \\
& \Gamma_{j}^{\prime}=\frac{\sqrt{u_{T}}}{t_{0}^{2}}\left[\mathrm{v}\left(t_{0}+t_{3}\right)+\sqrt{u_{L}}\left(t_{3} \mathrm{P}_{j}^{\prime \prime}+t_{0} \mathrm{P}_{j}^{\prime}\right)\right], \\
& t_{3}=3+u-2 \mathrm{v}, u_{T}=u \sin ^{2} \alpha, u_{L}=u \cos ^{2} \alpha,
\end{aligned}
$$

$$
\Gamma_{j}^{\prime \prime}=\frac{\sqrt{u_{T}}}{t_{0}}\left(\mathrm{v}+\sqrt{u_{L}} \mathrm{P}^{\prime \prime}\right)-s^{2} \frac{\sqrt{u_{T}}}{t_{0}^{2}}
$$

$$
\cdot\left[\mathrm{v} t_{3}+\sqrt{u_{L}} \mathrm{P}_{j}^{\prime \prime}(3-\mathrm{v})+\sqrt{u_{L}} \mathrm{P}_{j}^{\prime} t_{3}\right], u_{T}=u \sin ^{2} \alpha
$$

$u_{L}=u \cos ^{2} \alpha$, upper sign (index 1) corresponds to the ordinary wave; the lower sign (index 2 ) to the extraordinary wave.

Fluctuation of the phase of scattered electromagnetic wave caused by electron density fluctuations satisfying the boundary condition $\varphi_{1}\left(k_{x}, k_{y}, z=0\right)==0$ applying the modify smooth perturbation method $[6,7,9]$ normal to the direction of the principle plane has the following form:

$$
\begin{gathered}
\varphi_{1}(x, y, z)=i \frac{k_{0}}{2}\left(\tilde{Z}^{\prime}+i s \tilde{Z}^{\prime \prime}\right) \int_{-\infty}^{\infty} d k_{x} \int_{-\infty}^{\infty} d k_{y} n_{1}\left(k_{x}, k_{y}, z\right) . \\
\exp \left(i k_{x} x+i k_{y} y\right)
\end{gathered}
$$

$$
\begin{aligned}
& \cdot \exp \left(\frac { 1 } { 4 k _ { 0 } ^ { 2 } } \left\{\left[\tilde{d}_{2} \Gamma_{j}^{\prime \prime} k_{x}-\tilde{d}_{1}\left(2 k_{0}-s \Gamma_{j}^{\prime} k_{x}\right)\right]\right.\right. \\
& \left.\left.-i\left[\tilde{d}_{1} \Gamma_{j}^{\prime \prime} k_{x}+\tilde{d}_{2}\left(2 k_{0}-s \Gamma_{j}^{\prime} k_{x}\right)\right]\right\}(L-z)\right)
\end{aligned}
$$

where $\tilde{d}_{1}=k_{x}\left[k_{0} \Gamma_{j}^{\prime \prime}-\mathrm{P}_{j}^{\prime \prime}\left(k_{y}+k_{\perp}\right)\right]$,

$$
\begin{gathered}
\tilde{d}_{2}=k_{y}\left(k_{y}+2 k_{\perp}\right)+s k_{x}\left[\mathrm{P}_{j}^{\prime}\left(k_{y}+k_{\perp}\right)-\Gamma_{j}^{\prime} k_{0}\right], \\
\tilde{Z}^{\prime}=-\varepsilon_{0}\left[\left(1+\sqrt{u_{L}} \mathrm{P}_{j}^{\prime \prime}-\sqrt{u_{T}} \Gamma_{j}^{\prime \prime}\right)+\right. \\
\left.+2 s^{2}\left(\sqrt{u_{L}} \mathrm{P}_{j}^{\prime}-\sqrt{u_{T}} \Gamma_{j}^{\prime}\right)\right], \tilde{Z}^{\prime \prime}=-\varepsilon_{0}\left[(1+u)+\sqrt{u_{L}} .\right. \\
\left.\cdot\left(2 \mathrm{P}_{j}^{\prime \prime}-\mathrm{P}_{j}^{\prime}\right)+\sqrt{u_{T}}\left(\Gamma_{j}^{\prime}-2 \Gamma_{j}^{\prime \prime}\right)\right]
\end{gathered}
$$

Second order statistical moment of the phase fluctuations is:

$$
\begin{aligned}
& <\varphi_{1}(\mathbf{r}) \varphi_{1}(\mathbf{r}+\boldsymbol{\rho})>=-\frac{\pi}{2}(A+i B) k_{0}^{2} L \int_{-\infty}^{\infty} d k_{x} \int_{-\infty}^{\infty} d k_{y} \\
& \qquad \begin{array}{c}
V_{n}\left[k_{x}, k_{y}, \frac{1}{4 k_{0}^{2}}\left(i \Lambda_{1}-\Lambda_{2}\right)\right] \exp \left(-i k_{x} \rho_{x}-i k_{y} \rho_{y}\right), \\
\text { where: } A=\tilde{Z}^{\prime 2}-s^{2} \tilde{Z}^{\prime \prime 2}, B=2 s \tilde{Z}^{\prime} \tilde{Z}^{\prime \prime}, \\
\Lambda_{1}=-\frac{1}{4 k_{0}^{2}}\left[2 k_{0}\left(\mathrm{P}_{j}^{\prime \prime} k_{\perp}-\Gamma_{j}^{\prime \prime} k_{0}\right)+\Gamma_{j}^{\prime \prime} k_{y}^{2}\right] k_{x}+ \\
+s\left(\mathrm{P}_{j}^{\prime} \Gamma_{j}^{\prime \prime}-\mathrm{P}_{j}^{\prime \prime} \Gamma_{j}^{\prime}\right) k_{y} k_{x}^{2},
\end{array}
\end{aligned}
$$




$$
\begin{aligned}
& \Lambda_{2}=\frac{1}{4 k_{0}^{2}}\left\{4 k_{0} k_{\perp} k_{y}-\mathrm{P}_{j}^{\prime \prime} \Gamma_{j}^{\prime \prime} k_{y} k_{x}^{2}+\right. \\
& \left.+s\left[2 k_{0}\left(\mathrm{P}_{j}^{\prime} k_{\perp}-\Gamma_{j}^{\prime} k_{0}\right)-\Gamma_{j}^{\prime} k_{y}^{2}\right] k_{x}\right\},
\end{aligned}
$$

$V_{n}(\mathbf{k})$ is the arbitrary $3 \mathrm{D}$ spatial spectral function of elec-tron density fluctuations, the asterisk indicates the complex conjugate, $L$ is the mean distance between the observer and the plasma irregularities; $\rho_{x}$ and $\rho_{y}$ are the distances between observation points spaced apart in the principle and perpendicular planes, respectively.

The wave structure function of the phase fluctuations is [3]:

$$
D_{1}\left(\mathbf{r}_{1}, \mathbf{r}_{2}\right)=<\left(\varphi_{1}\left(\mathbf{r}_{1}\right)-\varphi_{1}\left(\mathbf{r}_{2}\right)\right)\left(\varphi_{1}^{*}\left(\mathbf{r}_{1}\right)-\varphi_{1}^{*}\left(\mathbf{r}_{2}\right)\right)>
$$

in the high-latitude polar ionospheric region $\left(\alpha=0^{0}\right)$ we have:

$$
\begin{gathered}
D_{1}\left(\rho_{x}, \rho_{y}, L\right)=\pi\left(Z^{\prime 2}+s^{2} Z^{\prime \prime 2}\right) k_{0}^{2} L \int_{-\infty}^{\infty} d k_{x} \int_{-\infty}^{\infty} d k_{y} \\
V_{n}\left\{k_{x}, k_{y},-\frac{1}{2 k_{0}}\left[\left(2 k_{\perp}+k_{y}\right) k_{y}+s \mathrm{P}_{j}^{\prime}\left(k_{\perp}+k_{y}\right) k_{x}\right]\right\} \\
\cdot\left[1-\cos \left(k_{x} \rho_{x}+k_{y} \rho_{y}\right)\right]
\end{gathered}
$$

Usually in scintillation studies, the fluctuations are characterized by the scintillation index whose properties can be related to those of the ionospheric irregularities by the application of the diffraction theory. For weak scattering the scintillation level $S_{4}$ is expressed via second order moments of the phase fluctuations [10]:

$$
S_{4}^{2}=2 \int_{-\infty}^{\infty} d k_{x} \int_{-\infty}^{\infty} d k_{y} W_{\varphi}\left(k_{x}, k_{y}, L\right) \sin ^{2}\left(\frac{k_{x}^{2}+k_{y}^{2}}{k_{f}^{2}}\right),
$$

where: $k_{f}=(4 \pi / \lambda z)^{1 / 2}$ is the Fresnel wavenumber. The sinusoidal term is responsible for oscillations in the scintillation spectrum.

If the satellite and/or the ionosphere moves relative to the receiver, temporal variations of both intensity and phase are recorded. We shall use the assumption of "frozen-in" irregularities. If rigid plasma irregularities are assumed to be moving in the $X$ direction with apparent velocity $V_{x}$ transverse to the line of the sight path, the power spectrum $P_{S}(v, L)$ is computed from the power spectrum [10]:

$$
P_{S}(v, L)=4 P_{\varphi}(v, L) \sin ^{2}\left(\frac{v}{v_{f}}\right)^{2}
$$

The Fresnel frequency $v_{f}=V_{x} /(\pi \lambda L)^{1 / 2}$ is directly pro portional to the drift velocity $V_{x}$ of plasma irregularities; $(\lambda L)^{1 / 2}$ is the Fresnel radius, the sinusoidal term is responsible for oscillations in the scintillation spectrum.

\section{Numerical Calculations}

Scintillation level and the power spectrum we investigate for two frequencies of an incident electromagnetic waves 3 $\mathrm{MHz}\left(k_{0}=6.28 \cdot 10^{-2} \mathrm{~m}^{-1}\right)$ and $40 \mathrm{MHz}\left(k_{0}=0.84 \mathrm{~m}^{-1}\right)$. An RH-560 rocket flight was conducted from Sriharikota rocket range (SHAR) to study electron density and electric field irregularities during spread F [11]. Large scale structures, with vertical scale sizes up to a few tens of $\mathrm{km}$, are seen at the altitude from $150 \mathrm{~km}$ to $257 \mathrm{~km}$. Small-scale structures having scale sizes of the order of few hundred meters are also seen, superimposed on large-scale structure in the entire region. A patch of very intense irregularities can be seen in 210-257 km region. Irregularities of a range of scale sizes starting from a few hundred meters to a few tens of kilometers are observed in this patch. Spaces receiver measurements made at Kingston, Jamaica, show [12] that irregularities causing the scintillation of signals from earth satellites are closely aligned along the magnetic field lines in the F-region, and the dip angle of the irregularities in the ionosphere is within $16^{0}$.

Numerical calculations are carried out for the irregularity model describing by the anisotropic Gaussian 3D correlation function of the electron density. Spectra of electron density fluctuations, scintillation measurements provide information about other irregularity parameters used in scintillation modeling: axial ratio along and across the magnetic field, orientation of plasma irregularities, slope of the in-situ spatial spectrum of the irregularities and the in-situ drift velocity of irregularities.

The anisotropic 3D Gaussian spectral function having certain mathematical advantages has the following form [4]:

$$
\begin{gathered}
V_{n}(\mathbf{k})=\sigma_{n}^{2} \frac{l_{\perp}^{2} l_{\|}}{8 \pi^{3 / 2}} \\
\exp \left(-\frac{k_{x}^{2} l_{\perp}^{2}}{4}-p_{1} \frac{k_{y}^{2} l_{\|}^{2}}{4}-p_{2} \frac{k_{z}^{2} l_{\|}^{2}}{4}-p_{3} k_{y} k_{z} l_{\|}^{2}\right),
\end{gathered}
$$

where: $p_{1}=\left(\sin ^{2} \gamma_{0}+\chi^{2} \cos ^{2} \gamma_{0}\right)^{-1}\left[1+\left(1-\chi^{2}\right)^{2}\right.$.

$$
\begin{aligned}
& \left.\cdot \sin ^{2} \gamma_{0} \cos ^{2} \gamma_{0} / \chi^{2}\right], p_{2}=\left(\sin ^{2} \gamma_{0}+\chi^{2} \cos ^{2} \gamma_{0}\right) \chi^{-2}, \\
& p_{3}=\left(1-\chi^{2}\right) \sin \gamma_{0} \cos \gamma_{0} / 2 \chi^{2}, \sigma_{n}^{2} \text { is the mean-square }
\end{aligned}
$$
fractional deviation of electron density. This spectral function contains parameter $\chi=l_{\|} / l_{\perp}$ (the ratio of longitudinal and transverse linear sizes of plasma irregularities) measuring the anisotropy of irregularities and the inclination angle $\gamma_{0}$ of elongated irregularities with respect to the external magnetic field. Anisotropy of the shape of irregularities is connected with the difference of the diffusion coefficients in the field 
align and field perpendicular directions.

Substituting (9) in the correlation function of the phase fluctuation we obtain:

$$
\begin{gathered}
W_{\varphi}\left(\eta_{x}, \eta_{y}, L\right)=\frac{\xi \Omega_{0}}{2 \sqrt{\pi} \chi} \int_{-\infty}^{\infty} d x \int_{-\infty}^{\infty} d y \exp \left(-i \eta_{x} x-i \eta_{y} y\right) \\
\quad \exp \left(-\frac{\xi^{2}}{4}\left\{\frac{p_{2}}{16} \Lambda_{0}^{2} x^{4}-s \frac{p_{2}}{8} \Lambda_{0} \Lambda_{4} x^{3}+\right.\right. \\
+\left[\frac{1}{\chi^{2}}+\left(\frac{p_{2}}{2} t-p_{3} y\right) \Lambda_{0}+s^{2} \frac{p_{2}}{16} \Lambda_{1}^{2}\right] x^{2}+ \\
\left.\left.-s\left(\frac{p_{2}}{2} t-p_{3} y\right) \Lambda_{1} x+\left(p_{1} y^{2}+p_{2} t^{2}-4 p_{3} t y\right)\right\}\right)
\end{gathered}
$$

where: $\Lambda_{0}=\Lambda_{3}-\left(\Gamma^{\prime \prime} \mathrm{P}^{\prime \prime}+s^{2} \Gamma^{\prime} \mathrm{P}^{\prime}\right) y, \eta_{x}=k_{0} \rho_{x}$,

$$
\begin{gathered}
\Lambda_{3}=\Gamma^{\prime \prime}\left(\Gamma^{\prime \prime}-\mathrm{P}^{\prime \prime} \mu\right)+s^{2} \Gamma^{\prime}\left(\Gamma^{\prime}-\mathrm{P}^{\prime} \mu\right), \eta_{y}=k_{0} \rho_{y}, \\
\Lambda_{4}=2\left(\Gamma^{\prime}-\mathrm{P}^{\prime} \mu\right)+2\left(\Gamma^{\prime} \mu-\mathrm{P}^{\prime}\right) y+\Gamma^{\prime} y^{2}
\end{gathered}
$$

$\Omega_{0}=\sigma_{n}^{2} \xi^{2} k_{0} L\left(\tilde{Z}^{\prime 2}+s^{2} \tilde{Z}^{\prime \prime 2}\right) / 8 \chi, \mu=k_{\perp} / k_{0}$ is the diffraction parameter, $t=(2 \mu+y) y / 2$. Parameters: $\mathrm{P}_{j}^{\prime}$, $\Gamma_{j}^{\prime}, \tilde{Z}^{\prime 2}$ and $\tilde{Z}^{\prime \prime 2}$ should be calculated from equations (3) and (4) for a given altitude of the ionosphere.

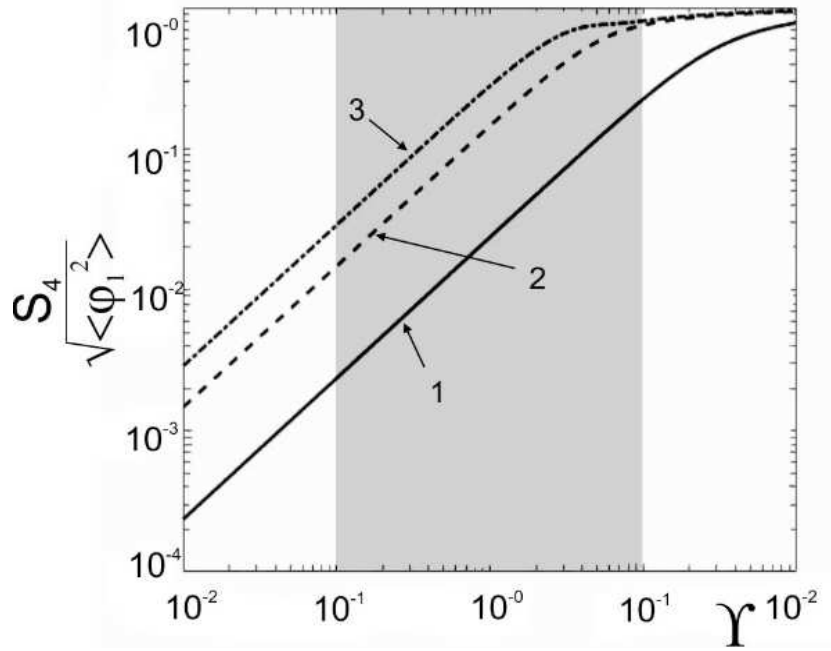

Figure 1. Normalized scintillation level as a function of distance below an irregular layer based on the Gaussian irregularities for $\xi=10$. Curve 1 corresponds to the isotropic case $\left(\chi=1, \gamma_{0}=0^{0}\right)$, curve $2(\chi=5$, $\left.\gamma_{0}=5^{0}\right)$, curve $3\left(\chi=12, \gamma_{0}=15^{0}\right)$.

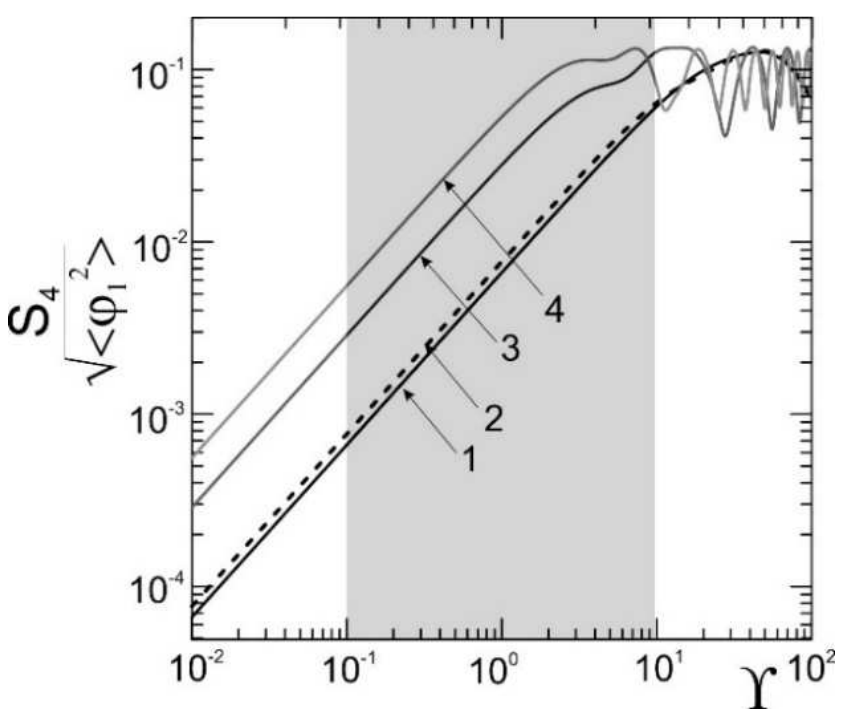

Figure 2. Normalized scintillation spectrum versus parameter $\Upsilon$ for $3 \mathrm{MHz}$ incident electromagnetic wave; one observation point $\eta_{x}=\eta_{y}=0$. Curves 1 and 2 correspond to the case: $\chi=10, \gamma_{0}=5^{0}$ and $10^{0}$, respectively; curves 3 and 4 - to the case: $\gamma_{0}=20^{0}, \chi=20$ and 30 , respectively.

Fig. 1 illustrates the dependence of the normalized scintillation level $S_{4} /\left\langle\varphi_{1}^{2}\right\rangle^{1 / 2}$ on the parameter $\Upsilon$ for the anisotropic Gaussian irregularities in the magnetized plasma at different parameters $\chi$ and $\gamma_{0} ; \eta_{x}=\eta_{y}=0$. The two asymptotic simplifications $\Upsilon \ll<1$ is associated with a significant filtering, non-fully developed diffraction pattern, whereas $\Upsilon>>1$ is associated with a fully developed pattern. Shaded area corresponds to the transition region between these two regions.

Fig. 2 represents the $\log -\log$ plots of the spectrum $S_{4} /\left\langle\varphi_{1}^{2}\right\rangle^{1 / 2}$ of scattered electromagnetic waves in the turbulent collision magnetized plasma versus parameter $\Upsilon$ perpendicular to the principle plane for different anisotropy parameter $\chi$ and the angle of inclination of anisotropic plasma irregularities with respect to the external magnetic field. Linear scale of irregularities is about $1 \mathrm{~km}(\xi=60)$, $s=0.01$. Numerical analyses show that in both cases: at fixed inclination angle increasing anisotropic parameter and at small parameters $\chi$ rising of the angle $\gamma_{0}$ not lead to the oscillations of the scintillation level. Since $\chi=13$ and $\gamma_{0}>15^{0}$ splashes of electromagnetic waves are observed at $\Upsilon=8$. Increasing anisotropic parameter $\chi$ up to 30 and at $\gamma_{0}=20^{\circ}$, after splash oscillations are appeared; at first they damped up to $\Upsilon>100$ and becomes stationary at $\Upsilon=800$. 


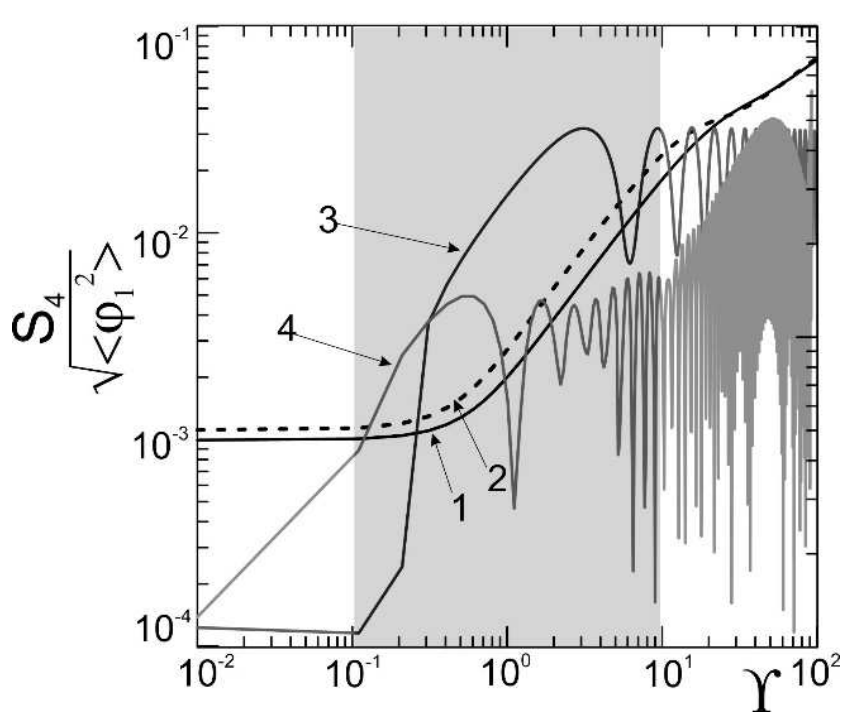

Figure 3. Normalized scintillation spectrum versus parameter $\Upsilon$ for $3 \mathrm{MHz}$ incident EM wave; observation points are at: $\eta_{x}=5, \eta_{y}=2$. Parameters of the curves 1-4 as in Figure 2.

The dependence of the log-log plots of the normalized scintillation spectrum $\left.S_{4} /<\varphi_{1}^{2}\right\rangle^{1 / 2}$ versus parameter $\Upsilon$ at $\eta_{x}=5$ and $\eta_{y}=2$ is presented on Figure 3. Numerical analyses show that at small anisotropic parameter $1 \leq \chi \leq 12$ electromagnetic waves fast dumped in the scintillation spectrum, $7 \leq \Upsilon \leq 50$. Increasing parameter since $\chi \geq 20$ and at the angle of inclination of elongated plasma irregularities with respect to the external magnetic field, $10^{0} \leq \gamma_{0} \leq 20^{0}$ undamped harmonic oscillations are generated in the polar region of the ionosphere. Numerical analyses show that at small anisotropic parameter

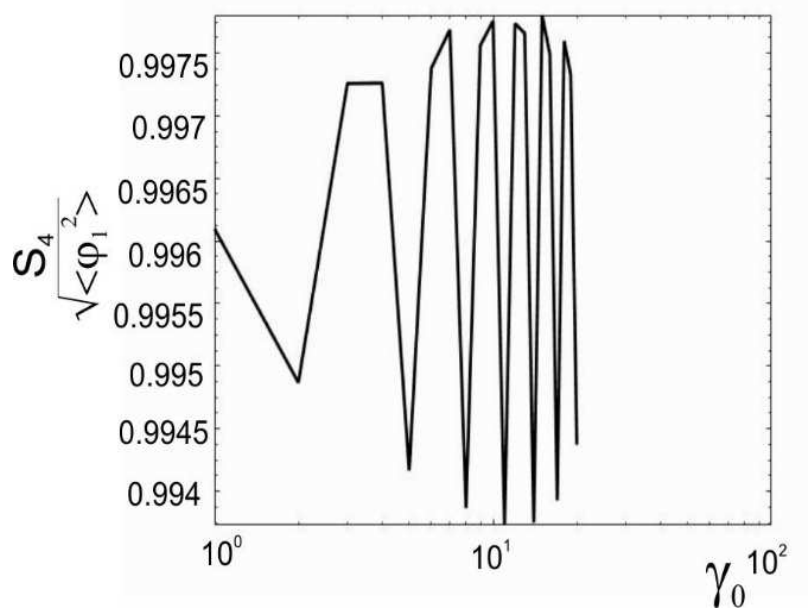

Figure 4. Normalized scintillation level as a function of the angle of inclination $\gamma_{0}$ at $\xi=5$.

$1 \leq \chi \leq 12$ electromagnetic waves fast dumped in the scintillation spectrum $7 \leq \Upsilon \leq 50$. Increasing parameter since $\chi \geq 20$ and at the angle of inclination of elongated plasma irregularities with respect to the external magnetic field, $10^{0} \leq \gamma_{0} \leq 20^{\circ}$ undamped harmonic oscillations are generated in the polar region of the ionosphere.

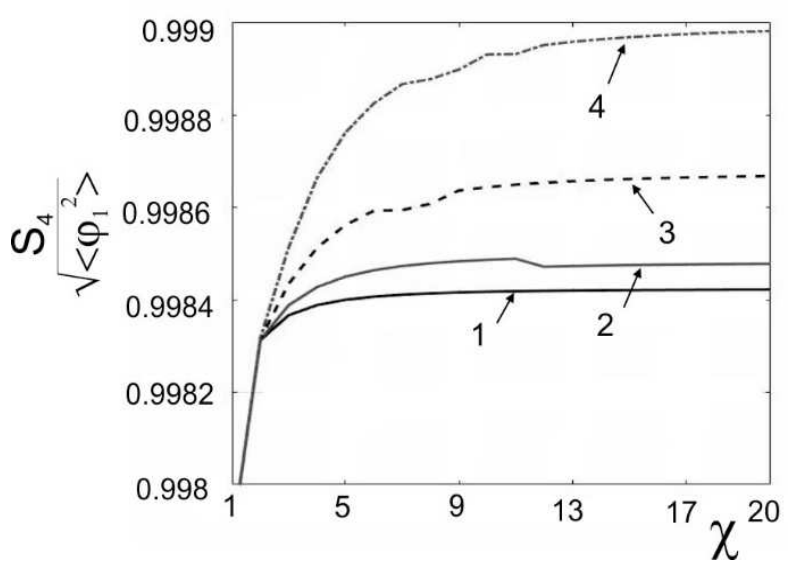

Figure 5. Normalized scintillation level as a function of the anisotropic factor $\chi$ at $\xi=5$ for $40 \mathrm{MHz}$ frequency incident electromagnetic wave. Culrve 1 corresponds to $\gamma_{0}=0^{0}$, curve $2-\gamma_{0}=5^{0}$, curve $3-\gamma_{0}=10^{0}$, curve 4 $\gamma_{0}=15^{0}$.

Fig. 4 illustrates the dependence of the function $\left.S_{4} /<\varphi_{1}^{2}\right\rangle^{1 / 2}$ on the inclination angle. Analyses show that maximums are at: $\gamma_{0}=1^{0}, 4^{0}, \ldots, 19^{0}$, minimums are at: $\gamma_{0}=2^{0}, 5^{0}, \ldots, 17^{0}$. Periodicity of maximums and minimums of scintillation is about three degrees. Analyses show that anisotropy factor of small-scale plasma irregularities $\left(l_{\|} \approx 80\right.$ $\mathrm{m})$ has no influence on the scintillation level of scattered radiation.

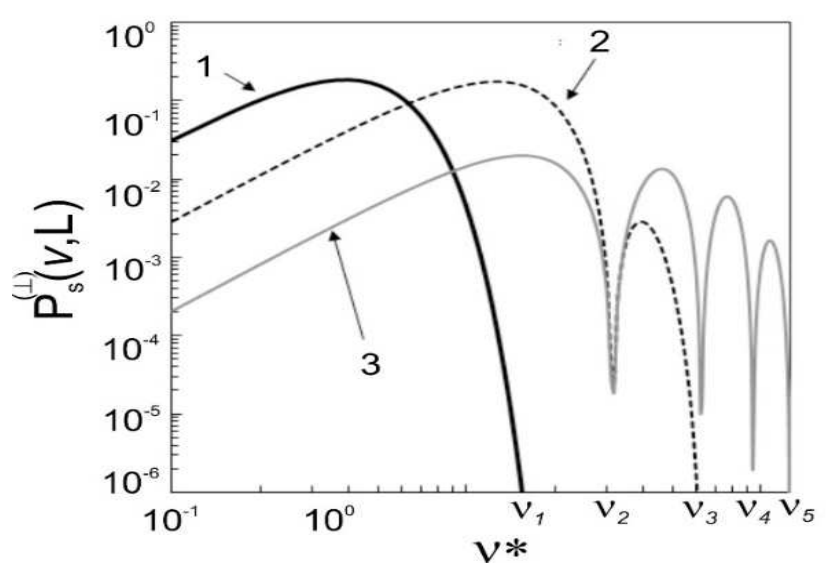

Figure 6. Power spectrum of the intensity fluctuations for the ordinary wave. Curve 1 corresponds to the isotropic case $\chi=1$ and $\gamma_{0}=0^{0}$, curve 2 depicts $\chi=5$ and $\gamma_{0}=5^{0}$, curve $3: \chi=15$ and $\gamma_{0}=10^{0} ; v_{*}=v / v_{0}$.

Fig. 5 represents the curves of the scintillation level versus anisotropy parameter $\chi$ for frequency $3 \mathrm{MHz}$ electromagnetic waves propagating in the turbulent magnetized collision plasma with small-scale irregularities 
$\left(l_{\|}=80 \mathrm{~m}\right)$ electron density fluctuations. In the polar ionosphere scintillations less sensitive to the geometrical parameters of plasma irregularities. Scintillations are more sensitive to the high-frequency electromagnetic waves increasing in proportion to the inclination angle of elongated irregularities with respect to the line of forces of an external magnetic field. At $\gamma_{0}=0^{0}$ the curves tend to the saturation starting from $\chi=8$, while at $\gamma_{0}=15^{0}$ from $\chi=23$.

The drift velocity varies in the interval $84 \mathrm{~m} / \mathrm{s}-102 \mathrm{~m} / \mathrm{s}$. If rigid plasma irregularities are moving along the $\mathrm{X}$ - axis, substituting (9) into equation (8) for high latitude polar ionosphere $\left(\alpha=0^{0}\right)$ we obtain:

$$
\begin{gathered}
P_{\varphi}^{(\perp)}(v, L)=\frac{\Omega_{0} \xi}{2 \sqrt{\pi} \chi v_{0}} \exp \left[-\frac{\xi^{2}}{4 \chi^{2}}\left(\frac{v}{v_{0}}\right)^{2}-i \eta_{x} \frac{v}{v_{0}}\right] \\
\int_{-\infty}^{\infty} d y \exp \left[-\frac{\xi^{2}}{4}\left(J_{1} \pm 3 s^{2} \frac{v}{v_{0}} e_{4}\right)-i \eta_{y} y\right],
\end{gathered}
$$

where: $v_{0}=k_{0} V_{x} / 2 \pi$,

$$
\begin{gathered}
e_{4}=\frac{p_{2}}{2} y^{3}+\left(\frac{3}{2} p_{2} \mu-2 p_{3}\right) y^{2}+\mu\left(p_{2} \mu-2 p_{3}\right) y, \\
J_{1}=\frac{p_{2}}{4} y^{4}+\left(p_{2} \mu-2 p_{3}\right) y^{3}+\left(p_{1}-4 p_{3} \mu+p_{2} \mu^{2}\right) y^{2} .
\end{gathered}
$$

Fig. 6 depicts curves of the power spectrum of the intensity fluctuations for both ordinary and extraordinary waves in the collision magnetized plasma perpendicular to the principle plane. Parameters: $\xi=10, \quad\left(l_{\|} \approx 160 \mathrm{~m}\right), \quad \eta_{x}=\eta_{y}=5$, $s=0.1$. Numerical calculations show that first minimum for the ordinary wave is at: $v_{1}=1.45 \mathrm{~Hz}$, next minimums are at: $3.2 \mathrm{~Hz} ; 6.3 \mathrm{~Hz} ; 9.4 \mathrm{~Hz} ; 12.9 \mathrm{~Hz}$. For the extraordinary wave first minimum is at $v_{1}=1.6 \mathrm{~Hz}$, next minimums are at: $3.2 \mathrm{~Hz}$, $6.1 \mathrm{~Hz}, 9,4 \mathrm{~Hz}, 12.6 \mathrm{~Hz}$. Frequency of both waves satisfy the condition: $1: 2: 3: 4$ and so on. Inflection points approximately are the same for both waves. Particularly for the curve 1: $v=0.5 \mathrm{~Hz}$; curve 2: $v=2 \mathrm{~Hz}$ and $4 \mathrm{~Hz}$; curve 3: $v=2 \mathrm{~Hz}, 5 \mathrm{~Hz}, 8 \mathrm{~Hz}, 11 \mathrm{~Hz}$ and $14 \mathrm{~Hz}$.

$8 \mathrm{~Hz}, 11 \mathrm{~Hz}$ and $14 \mathrm{~Hz}$.

\section{Conclusion}

Statistical characteristics of scattered electromagnetic wave have been obtained for arbitrary correlation function of electron density fluctuations and the angle between the wave vector of an incident wave and the direction of line of force of an external magnetic field. Analytical calculations were carried out for the anisotropic Gaussian correlation function of electron density fluctuations containing the anisotropy factor of elongated plasma irregularities and the angle of inclination of the irregularities to the external magnetic field. Numerical analyses were carried out for $3 \mathrm{MHz}$ incident wave for the high latitude ionosphere. Analyses of the normalized scintillation level of scattered radiation show that stationary oscillations are generated in the collision magnetized plasma (at the collision frequency $s \approx 0.01$ ) with small-scale plasma irregularities having characteristic spatial scale $80 \mathrm{~m}$. Scintillation spectrum reaches maximums and minimums with periodicity 3 degree substantially depending on the frequency of an incident wave. Scintillation level increases in proportion to the inclination angle of elongated irregularities with respect to the line of forces of an external magnetic field. At the frequency 40 $\mathrm{MHz}$ the curves of the normalized scintillation level tend to the saturation at big anisotropy factors in proportion of the slope angle, while at the frequency of an incident wave 3 $\mathrm{MHz}$ scintillation level less sensitive to the geometric parameters of the ionospheric plasma irregularities. Minimums of the power spectrum of the intensity fluctuations for both ordinary and extraordinary waves satisfy the condition: $1: 2: 3: 4$ and so on. Inflection points approximately are the same for both waves.

\section{Acknowledgements}

The work has been supported by the International Science and Technology Center (ISTC) under Grant \# G-2126 and Shota Rustaveli National Science Foundation under Grant \# FR/3/9-190/14.

\section{References}

[1] J. Aarons, "Global Morphology of Ionospheric Scintillation," Proc. IEEE, vol. 70, pp. 360-378, 1982.

[2] K. C. Yeh and C. H. Liu, "Radio Wave Scintillations in the Ionosphere,” Proc. IEEE, vol. 70, pp.324-360, 1982.

[3] A. Ishimaru, Wave Propagation and Scattering in Random Media, Vol. 2, Multiple Scattering, Turbulence, Rough Surfaces and Remote Sensing," IEEE Press, Piscataway, New Jersey, USA, 1997.

[4] G. V. Jandieri, A. Ishimaru, V. G. Jandieri, A. G. Khantadze and Zh. M. Diasamidze, "Model Computations of Angular Power Spectra for Anisotropic Absorptive Turbulent Magnetized Plasma," PIER, vol. 70, pp. 307-328, 2007.

[5] G. V. Jandieri, Zh. M. Diasamidze and M. R. Diasamidze, "Scintillation Spectra of Scattered Electromagnetic Waves in Turbulent Magnetized Plasma," Journal of Basic and Applied Physics, vol. 2, pp. 224-234, 2013.

[6] G. V. Jandieri, „Double-Humped Effect“ in the Turbulent Collision Magnetized Plasma“, PIER M, vol. 48, pp. 95-102, 2016.

[7] G. V. Jandieri, A. Ishimaru, B. S. Rawat and N. K. Tugushi, "Peculiarities of the Spatial Power Spectrum of Scattered Electromagnetic Waves in the Turbulent Collision Magnetized Plasma," PIER, vol. 152, pp. 137-149, 2015.

[8] V. L. Ginzburg, "Propagation of Electromagnetic Waves in Plasma," Gordon and Beach, New York. 1961. 
[9] G. V. Jandieri, A. Ishimaru, N. F. Mchedlishvili and I. G. Takidze, "Spatial Power Spectrum of Multiple Scattered Ordinary and Extraordinary Waves in Magnetized Plasma with Electron Density Fluctuations," PIER (M)), vol. 25, pp. 87-100, 2012.

[10] C. L. Rufenach, "Power-law Wavenumber Spectrum Deduced from Ionospheric Scintillation Observations," Journal of Geophysical Research, vol. 77, pp. 4761-4772, 1972.

[11] S. Raizada, and H. S. S. Sinha, "Some new features of electron density irregularities over SHAR during strong spread F," Ann. Geophysicae, vol. 18, pp. 141-151, 2000.
[12] A. A. Chen, and G. S. Kent, "Determination of the orientation of ionospheric irregularities causing scintillation of signal from earth satellites," Journal of Atmospheric and Terrestrial Physics, vol. 34, pp. 1411-1414, 1972.

[13] T. Farges, and H. F. Blanc, "HF radar observations of irregularities in the daytime equatorial $\mathrm{F}$ region," Journal of Atmospheric and Terrestrial Physics, vol. 64, pp. 1565-1571, 2002. 